\title{
Strongly $\lambda$-statistically and strongly Valle-Poussin pre-Cauchy sequences in probabilistic metric spaces
}

\author{
Argha Ghosh and Samiran Das
}

\begin{abstract}
We introduce the notions of strongly $\lambda$-statistically pre-Cauchy and strongly Valle-Poussin pre-Cauchy sequences in probabilistic metric spaces endowed with strong topology. And we show that these two new notions are equivalent. Strongly $\lambda$-statistically convergent sequences are strongly $\lambda$-statistically pre-Cauchy sequences, and we give an example to show that there is a sequence in a probabilistic metric space which is strongly $\lambda$-statistically pre-Cauchy but not strongly $\lambda$-statistically convergent.
\end{abstract}

Keywords. Probabilistic metric space, strongly $\lambda$-statistical convergence, strongly $\lambda$-statistical pre-Cauchy, strongly statistical pre-Cauchy, strongly Valle-Poussin pre-Cauchy

\section{Introduction:}

Throughout the article, $\lambda$ will always denote a non-decreasing sequence of positive real numbers tending to $\infty$ such that $\lambda_{1}=1, \lambda_{n+1} \leq \lambda_{n}+1, n \in \mathbb{N}$. And we write $\Delta_{\infty}$ to denote all such sequences $\lambda$.

In 1951, in order to study sequences of real numbers similar in some sense to the convergent sequences of real numbers, Fast [4] and Schoenberg [10] independently introduced the notion of statistical convergence of sequences of real numbers. Afterward, in [1], in order to provide a Cauchy-like criterion for the statistical convergent sequences of real numbers, Connor et al. introduced the notion of pre-Cauchy sequences of real numbers and established a relationship between the notions of statistical convergence and pre-Cauchy sequences of real numbers. In 2000, Mursaleen [8] introduced the notion of $\lambda$-statistical convergence of sequences of real numbers and established its relationship with the notion of statistical convergence of sequences of real numbers. For a survey on this direction, see $[2,3,5]$ and many others.

On the other hand, the concept of Probabilistic metric spaces was introduced by Menger [7] under the name of statistical metric spaces. In this theory, the distance between two points $u, v$ is a distribution function $F_{u v}$. And the value of the function $F_{u v}$ at any $t>0$, that is, $F_{u v}(t)$ can be interpreted as the probability that the distance between $u$ and $v$ is less than $t$. After Menger, the theory of probabilistic metric spaces was studied and developed by Schwiezer and

Received date: September 13, 2020; Published online: April 11, 2021.

2010 Mathematics Subject Classification. 54E70, 40B05.

Corresponding author: Argha Ghosh. 
Sklar [12, 13, 14], Tardiff [15], Thorp [16] and many others. For a survey on this direction, see the book written by Schwiezer and Sklar [14].

One can define several topologies on this space, however, strong topology is the only one which is getting more attention over the years. In [11], Şençimen et al. remarked that the strong topology is first countable and Hausdorff, it can be characterized in terms of strong convergence of sequences. Moreover, to provide a more general framework for applications, Şençimen et al. [11] extended the notion of strong convergence of sequences to the notion of strong statistical convergence of sequences. Recently, Malik and Das [6] studied the notion of strong $\lambda$-statistical convergence of sequences which is a generalization of the notion of strong statistical convergence of sequences.

In point of view of recent applications of probabilistic metric spaces, in this article, we provide a Cauchy-like criterion of strong statistical and strong $\lambda$-statistical convergence of sequences in probabilistic metric spaces. We introduce the notion of strongly $\lambda$-statistically preCauchy sequences in probabilistic metric spaces. Strongly $\lambda$-statistically convergent sequences are strongly $\lambda$-statistically pre-Cauchy sequences, and we give an example to show that there is a sequence in a probabilistic metric space which is strongly $\lambda$-statistically pre-Cauchy but not strongly $\lambda$-statistically convergent. Therefore, in the realm of PM spaces, our notion resolved the question: can one decide if a sequence is strongly $\lambda$-statistically convergent without knowing the strong $\lambda$-statistical limit of the sequence in advance? Further, this notion provides a tool for the applications of the strong topology on the PM spaces. Also, we introduce the notion of strongly Valle-Poussin pre-Cauchy sequences to understand the notion of strongly $\lambda$-statistically pre-Cauchy sequences in probabilistic metric spaces differently. And these two new notions are equivalent.

\section{Preliminaries}

First, we familiarize reader with the basic concepts of statistical convergence, statistical pre-Cauchy and $\lambda$-statistical convergence of sequences of real numbers.

The notion of asymptotic density of the subsets of the set of all natural numbers $\mathbb{N}$ plays a central role in the concept of statistical convergence of sequences. We recall that a set $A \subset \mathbb{N}$ is said to have asymptotic density $d(A)$ if

$$
d(A)=\lim _{n \rightarrow \infty} \frac{|A(n)|}{n},
$$

where for all $n \in \mathbb{N}, A(n)=\{k \in A: k \leq n\}$.

Definition 1. [5] A sequence $\left\{x_{k}\right\}_{k \in \mathbb{N}}$ of real numbers is said to be statistically convergent to $l \in \mathbb{R}$ if, for every $\varepsilon>0, d(A(\varepsilon))=0$, where $A(\varepsilon)=\left\{k \in \mathbb{N}:\left|x_{k}-l\right| \geq \varepsilon\right\}$.

Definition 2. [1] A sequence $\left\{x_{k}\right\}_{k \in \mathbb{N}}$ of real numbers is said to be statistically pre-Cauchy if, for every $\varepsilon>0$,

$$
\lim _{n \rightarrow \infty} \frac{1}{n^{2}}\left|\left\{(j, k) \in[1, n] \times[1, n]:\left|x_{j}-x_{k}\right| \geq \varepsilon\right\}\right|=0
$$

From now on rest of the article, for each $n \in \mathbb{N}$, we write $I_{n}=\left[n-\lambda_{n}+1, n\right]$, where $\lambda=\left\{\lambda_{n}\right\} \in \Delta_{\infty}$. 
Definition 3. [8] A sequence $\left\{x_{k}\right\}_{k \in \mathbb{N}}$ of real numbers is said to be $\lambda$-statistically convergent to $l \in \mathbb{R}$ if, for every $\varepsilon>0$,

$$
\lim _{n \rightarrow \infty} \frac{1}{\lambda_{n}}\left|\left\{k \in I_{n}:\left|x_{k}-l\right| \geq \varepsilon\right\}\right|=0
$$

In 2000, Mursaleen [8] defined the notion of the generalized de la Valle-Poussin mean for a sequence $x=\left\{x_{k}\right\}$ by the the formula $\frac{1}{\lambda_{n}} \sum_{k \in I_{n}} x_{k}$. Note that whenever $\lambda_{n}=n$ we have the notion of Cesro mean for the sequence.

Definition 4. [8] A sequence $\left\{x_{k}\right\}_{k \in \mathbb{N}}$ of real numbers is said to be strongly $(V, \lambda)$-summable to $l \in \mathbb{R}$ if,

$$
\lim _{n \rightarrow \infty} \frac{1}{\lambda_{n}} \sum_{k \in I_{n}}\left|x_{k}-l\right|=0 .
$$

Theorem 2.1. [8] For bounded sequences of real numbers, the notions of $\lambda$-statistical convergence and strong $(V, \lambda)$-summability are equivalent.

Now we recall some basic ideas related to the probabilistic metric spaces (briefly PM spaces) (see $[12,13,14]$ and many others).

Definition 5. A non decreasing function $f:[-\infty, \infty] \rightarrow[0,1]$ with $f(-\infty)=0$ and $f(\infty)=1$ is called a distribution function.

We denote the set of all left continuous distribution functions over $(-\infty, \infty)$ by $\mathcal{D}$.

We consider a partial relation $\leq$ on $\mathcal{D}$ defined by $g \leq f$ if and only if $g(x) \leq f(x)$ for all $x \in[-\infty, \infty]$.

Definition 6. For any $q \in[-\infty, \infty]$ the unit step at $q$ is denoted by $\epsilon_{q}$ and is defined to be a function in $\mathcal{D}$ given by

$$
\begin{aligned}
\epsilon_{q}(t) & =0, \quad-\infty \leq t \leq q \\
& =1, \quad q<t \leq \infty
\end{aligned}
$$

In particular,

$$
\begin{aligned}
\epsilon_{0}(t) & =0, \quad-\infty \leq t \leq 0 \\
& =1, \quad 0<t \leq \infty
\end{aligned}
$$

Definition 7. A sequence $\left\{f_{n}\right\}_{n \in \mathbb{N}}$ of distribution functions is said to converge weakly to a distribution function $f$, if the sequence $\left\{f_{n}(t)\right\}_{n \in \mathbb{N}}$ converges to $f(t)$ at each continuity point $t$ of $f$. In this case, we write $f_{n} \stackrel{w}{\rightarrow} f$.

Definition 8. The distance between $f$ and $g$ in $\mathcal{D}$ is denoted by $d_{L}(f, g)$ and is defined to be the infimum of all numbers $h \in(0,1]$ such that the inequalities

$$
\begin{aligned}
f(t-h)-h \leq g(t) & \leq f(t+h)+h \\
\text { and } g(t-h)-h \leq f(t) & \leq g(t+h)+h
\end{aligned}
$$

hold for all $t \in\left(-\frac{1}{h}, \frac{1}{h}\right)$. 
It is known from the literature that $d_{L}$ is a metric on $\mathcal{D}$ and for any sequence $\left\{f_{n}\right\}_{n \in \mathbb{N}}$ in $\mathcal{D}$ and $f \in \mathcal{D}$, we have

$$
f_{n} \stackrel{w}{\rightarrow} f \text { if and only if } d_{L}\left(f_{n}, f\right) \rightarrow 0 \text {. }
$$

Definition 9. A non-decreasing real valued function $h$ defined on $[0, \infty]$ that satisfies $h(0)=0$ and $h(\infty)=1$ and is left continuous on $(0, \infty)$ is called a distance distribution function (d.d.f. in short).

The set of all distance distribution functions is denoted by $\mathcal{D}^{+}$. Moreover, the function $d_{L}$ is a metric on $\mathcal{D}^{+}$.

Theorem 2.2. [14] Let $f \in \mathcal{D}^{+}$be given. Then for any $t>0, f(t)>1-t$ if and only if $d_{L}\left(f, \epsilon_{0}\right)<t$.

Definition 10. A triangle function is a binary operation $\tau$ on $\mathcal{D}^{+}$, which is commutative, nondecreasing, associative in each place and $\epsilon_{0}$ is the identity.

Definition 11. A probabilistic metric space, briefly $\mathrm{PM}$ space, is a triplet $(P, F, \tau)$ where $P$ is a nonempty set whose elements are the points of the space; $F$ is a function from $P \times P$ into $\mathcal{D}^{+}$, $\tau$ is a triangle function, and the following conditions are satisfied for all $x, y, z \in P$ :

1. $F(x, x)=\epsilon_{0}$

2. $F(x, y) \neq \epsilon_{0}$ if $x \neq y$

3. $F(x, y)=F(y, x)$

4. $F(x, z) \geq \tau(F(x, y), F(y, z))$.

From now on we denote $F(x, y)$ by $F_{x y}$ and its value at $t$ by $F_{x y}(t)$.

Example 1. Let $f \in \mathcal{D}^{+}$be fixed and distinct from $\epsilon_{0}$ and $\epsilon_{\infty}$. Then we have the equilateral PM space $(P, F, M)$ where $F$ is defined by

$$
F_{p q}=\left\{\begin{array}{lll}
f & ; & \text { if } p \neq q \\
\epsilon_{0} & ; & \text { if } p=q
\end{array}\right.
$$

and $M$ is the maximal triangle function.

Definition 12. Let $(P, F, \tau)$ be a PM space. For $x \in P$ and $r>0$, the strong $r$-neighborhood of $x$ is denoted by $\mathcal{N}_{x}(r)$ and is defined by

$$
\mathcal{N}_{x}(r)=\left\{y \in P: F_{x y}(r)>1-r\right\}
$$

In this case, the collection $\mathfrak{N}_{x}=\left\{\mathcal{N}_{x}(r): r>0\right\}$ is said to be the strong neighborhood system at $x$ and the union $\mathfrak{N}=\bigcup_{x \in P} \mathfrak{N}_{x}$ is said to be the strong neighborhood system for $P$.

From the Theorem 2.2, we have $\mathcal{N}_{x}(r)=\left\{y \in P: d_{L}\left(F_{x y}, \epsilon_{0}\right)<r\right\}$. If $\tau$ is continuous, then the strong neighborhood system $\mathfrak{N}$ determines a Hausdorff topology for $P$. This topology is said to be the strong topology for $P$.

Definition 13. Let $(P, F, \tau)$ be a PM space. Then for any $r>0$, the subset $\mathfrak{V}(r)$ of $P \times P$ given by 


$$
\mathfrak{V}(r)=\left\{(x, y): F_{x y}(r)>1-r\right\}
$$

is said to be the strong $r$-vicinity.

Theorem 2.3. [14] Let $(P, F, \tau)$ be a PM space and $\tau$ be continuous. Then for any $r>0$, there is an $s>0$ such that $\mathfrak{V}(s) \circ \mathfrak{V}(s) \subset \mathfrak{V}(r)$, where $\mathfrak{V}(s) \circ \mathfrak{V}(s)=\{(x, z)$ : for some $y,(x, y)$ and $(y, z) \in \mathfrak{V}(s)\}$.

Note 1. By the virtue of the hypothesis of the Theorem 2.3, we can say, for any $r>0$, there is an $s>0$ such that $F_{a b}(r)>1-r$ whenever $F_{a c}(s)>1-s$ and $F_{c b}(s)>1-s$. Equivalently, we can write for any $r>0$, there is an $s>0$ such that $d_{L}\left(F_{a b}, \epsilon_{0}\right)<r$ whenever $d_{L}\left(F_{a c}, \epsilon_{0}\right)<s$ and $d_{L}\left(F_{c b}, \epsilon_{0}\right)<s$.

From now on throughout this work, $P$ will always denote the PM space $(P, F, \tau)$ endowed with the strong topology.

Definition 14. [11] Let $(P, F, \tau)$ be a PM space. A sequence $x=\left\{x_{k}\right\}_{k \in \mathbb{N}}$ in $P$ is said to be strongly convergent to $l \in P$ if, for every $t>0, \exists$ a natural number $k_{0}$ such that

$$
x_{k} \in \mathcal{N}_{l}(t) \quad \text { whenever } k \geq k_{0} .
$$

In this case, we write $F-\lim _{k \rightarrow \infty} x_{k}=l$ or $x_{k} \stackrel{F}{\longrightarrow} l$.

Definition 15. [11] Let $(P, F, \tau)$ be a PM space. A sequence $x=\left\{x_{k}\right\}_{k \in \mathbb{N}}$ in $P$ is said to be strongly statistically convergent to $l \in P$ if, for any $t>0$

$$
d\left(\left\{k \in \mathbb{N}: F_{x_{k} l}(t) \leq 1-t\right\}\right)=0 \quad \text { or } \quad d\left(\left\{k \in \mathbb{N}: x_{k} \notin \mathcal{N}_{l}(t)\right\}\right)=0 .
$$

In this case, we write $s t^{F}-\lim _{k \rightarrow \infty} x_{k}=l$.

Definition 16. [6] A sequence $x=\left\{x_{k}\right\}_{k \in \mathbb{N}}$ in a PM space $(P, F, \tau)$ is said to be strongly $\lambda$-statistically convergent to $l \in P$ if, for every $t>0$,

$$
\lim _{n \rightarrow \infty} \frac{1}{\lambda_{n}}\left|\left\{k \in I_{n}: F_{x_{k} l}(t) \leq 1-t\right\}\right|=0 .
$$

or

$$
\lim _{n \rightarrow \infty} \frac{1}{\lambda_{n}}\left|\left\{k \in I_{n}: x_{k} \notin \mathcal{N}_{l}(t)\right\}\right|=0 .
$$

In this case, we write $s t_{\lambda}^{F}-\lim _{k \rightarrow \infty} x_{k}=l$ or simply as $x_{k} \stackrel{s t_{\lambda}^{F}}{\longrightarrow} l$.

\section{Strongly $\lambda$-statistical pre-Cauchy}

In this section, we introduce the notion of strongly $\lambda$-statistically pre-Cauchy and establish a Cauchy like criterion for a strong $\lambda$-statistically convergent sequence in probabilistic metric spaces.

Definition 17. A sequence $x=\left\{x_{k}\right\}_{k \in \mathbb{N}}$ in a PM space $(P, F, \tau)$ is said to be strongly $\lambda$ statistically pre-Cauchy if for every $t>0$,

$$
\lim _{n \rightarrow \infty} \frac{1}{\lambda_{n}^{2}}\left|\left\{(j, k) \in I_{n} \times I_{n}: F_{x_{j} x_{k}}(t) \leq 1-t\right\}\right|=0 .
$$


Note 2. In the above Definition 17 , if we replace $\lambda_{n}$ by $n$ for each $n \in \mathbb{N}$, then we have the notion of strong statistically pre-Cauchy.

Theorem 3.1. If $\liminf _{n \rightarrow \infty} \frac{\lambda_{n}}{n}>0$, then the notion of strongly statistically pre-Cauchy implies the notion of strongly $\lambda$-statistically pre-Cauchy.

Proof. Let $x=\left\{x_{k}\right\}_{k \in \mathbb{N}}$ be a sequence in a PM space $(P, F, \tau)$ and $x$ is strongly statistically pre-Cauchy. Let $t>0$ be given. Then for $t>0$, we have

$$
\begin{aligned}
& \frac{1}{n^{2}}\left|\left\{(j, k) \in[1, n] \times[1, n]: F_{x_{j} x_{k}}(t) \leq 1-t\right\}\right| \\
\geq & \frac{1}{n^{2}}\left|\left\{(j, k) \in I_{n} \times I_{n}: F_{x_{j} x_{k}}(t) \leq 1-t\right\}\right| \\
= & \frac{\lambda_{n}^{2}}{n^{2}} \frac{1}{\lambda_{n}^{2}}\left|\left\{(j, k) \in I_{n} \times I_{n}: F_{x_{j} x_{k}}(t) \leq 1-t\right\}\right| .
\end{aligned}
$$

Now $\left\{\lambda_{n}\right\}_{n \in \mathbb{N}}$ is a non-decreasing sequence of positive numbers such that $\lambda_{1}=1, \lambda_{n+1} \leq \lambda_{n}+1$, $\lambda_{n} \leq n$ for all $n$ and hence the quotient $\frac{\lambda_{n}}{n}$ is nonnegative and bounded above by 1 . So we have $0<\liminf _{n \rightarrow \infty} \frac{\lambda_{n}^{2}}{n^{2}} \leq 1$ and since $\lim _{n \rightarrow \infty} \frac{1}{n^{2}}\left|\left\{(j, k) \in[1, n] \times[1, n]: F_{x_{j} x_{k}}(t) \leq 1-t\right\}\right|=0$, so

$$
\lim _{n \rightarrow \infty} \frac{1}{\lambda_{n}^{2}}\left|\left\{(j, k) \in I_{n} \times I_{n}: F_{x_{j} x_{k}}(t) \leq 1-t\right\}\right|=0 .
$$

Hence $x$ is strongly $\lambda$-statistically pre-Cauchy.

Theorem 3.2. Let $(P, F, \tau)$ be a PM space. If a sequence $x=\left\{x_{k}\right\}_{k \in \mathbb{N}}$ is strongly $\lambda$-statistically convergent, then it is strongly $\lambda$-statistically pre-Cauchy in $P$.

Proof. Let the sequence $x=\left\{x_{k}\right\}_{k \in \mathbb{N}}$ be strongly $\lambda$-statistically convergent to $l \in P$. Let $t>0$ be given. Then there exists $s>0$ such that $d_{L}\left(F_{u w}, \epsilon_{0}\right)<t$ whenever $d_{L}\left(F_{u v}, \epsilon_{0}\right)<s$, $d_{L}\left(F_{v w}, \epsilon_{0}\right)<s$ and $u, v, w \in P$. Let

$$
E_{n}=\left\{k \in I_{n}: F_{x_{k} l}(s) \leq 1-s\right\} .
$$

Then $\lim _{n \rightarrow \infty} \frac{\left|E_{n}\right|}{\lambda_{n}}=0$. Now let $F_{n}=I_{n} \backslash E_{n}$. Then $\lim _{n \rightarrow \infty} \frac{\left|F_{n}\right|}{\lambda_{n}}=1$. Then for $j, k \in F_{n}$, we have $F_{x_{j} l}(s)>1-s$ and $F_{x_{k} l}(s)>1-s$, that is, $d_{L}\left(F_{x_{j} l}, \epsilon_{0}\right)<s$ and $d_{L}\left(F_{x_{k} l}, \epsilon_{0}\right)<s$. Thus for $j, k \in F_{n}$, we have $d_{L}\left(F_{x_{j} x_{k}}, \epsilon_{0}\right)<t$, that is, $F_{x_{j} x_{k}}(t)>1-t$. Hence

$$
F_{n} \times F_{n} \subset\left\{(j, k) \in I_{n} \times I_{n}: F_{x_{j} x_{k}}(t)>1-t\right\} .
$$

This implies

$$
\left[\frac{\left|F_{n}\right|}{\lambda_{n}}\right]^{2} \leq \frac{1}{\lambda_{n}^{2}}\left|\left\{(j, k) \in I_{n} \times I_{n}: F_{x_{j} x_{k}}(t)>1-t\right\}\right| .
$$

Since $\lim _{n \rightarrow \infty} \frac{\left|F_{n}\right|}{\lambda_{n}}=1$, so

$$
\lim _{n \rightarrow \infty} \frac{1}{\lambda_{n}^{2}}\left|\left\{(j, k) \in I_{n} \times I_{n}: F_{x_{j} x_{k}}(t)>1-t\right\}\right|=1 .
$$

Thus

$$
\lim _{n \rightarrow \infty} \frac{1}{\lambda_{n}^{2}}\left|\left\{(j, k) \in I_{n} \times I_{n}: F_{x_{j} x_{k}}(t) \leq 1-t\right\}\right|=0 .
$$

Hence the sequence $x$ is strongly $\lambda$-statistically pre-Cauchy in $P$. 
Now we state a necessary and sufficient condition for a sequence to be strongly $\lambda$-statistically pre-Cauchy in a probabilistic metric space.

Theorem 3.3. Let $(P, F, \tau)$ be a $P M$ space and $x=\left\{x_{k}\right\}_{k \in \mathbb{N}}$ be a sequence in $P$. Then the sequence $x$ is strongly $\lambda$-statistically pre-Cauchy if and only if

$$
\lim _{n \rightarrow \infty} \frac{1}{\lambda_{n}^{2}} \sum_{j, k \in I_{n}} d_{L}\left(F_{x_{j} x_{k}}, \epsilon_{0}\right)=0 .
$$

Proof. At first, we assume that

$$
\lim _{n \rightarrow \infty} \frac{1}{\lambda_{n}^{2}} \sum_{j, k \in I_{n}} d_{L}\left(F_{x_{j} x_{k}}, \epsilon_{0}\right)=0 .
$$

Let $t>0$ be given. Since $\lambda_{n}>0$ for all $n \in \mathbb{N}$ and $t>0$, so we have

$$
\begin{aligned}
& \frac{1}{\lambda_{n}^{2}} \sum_{j, k \in I_{n}} d_{L}\left(F_{x_{j} x_{k}}, \epsilon_{0}\right) \\
\geq & t\left(\frac{1}{\lambda_{n}^{2}}\left|\left\{(j, k) \in I_{n} \times I_{n}: d_{L}\left(F_{x_{j} x_{k}}, \epsilon_{0}\right) \geq t\right\}\right|\right) \geq 0 .
\end{aligned}
$$

Thus from the squeeze lemma for limits, we have

$$
\lim _{n \rightarrow \infty} \frac{1}{\lambda_{n}^{2}}\left|\left\{(j, k) \in I_{n} \times I_{n}: d_{L}\left(F_{x_{j} x_{k}}, \epsilon_{0}\right) \geq t\right\}\right|=0 .
$$

In other words,

$$
\lim _{n \rightarrow \infty} \frac{1}{\lambda_{n}^{2}}\left|\left\{(j, k) \in I_{n} \times I_{n}: F_{x_{j} x_{k}}(t) \leq 1-t\right\}\right|=0 .
$$

Hence the sequence $x$ is strongly $\lambda$-statistically pre-Cauchy in $P$.

Conversely, we assume that $x$ is strong $\lambda$-statistically pre-Cauchy sequence in $P$. Let $t>0$ be given. Choose $s>0$ and $t_{0}>0$ so that $\frac{s}{2}+t_{0}<t$. Since $d_{L}\left(F_{p q}, \epsilon_{0}\right) \leq 1$ for every $p, q \in P$ and $\lambda_{n}>0$ for $n \in \mathbb{N}$, so we have

$$
\begin{aligned}
& \frac{1}{\lambda_{n}^{2}} \sum_{j, k \in I_{n}} d_{L}\left(F_{x_{j} x_{k}}, \epsilon_{0}\right)
\end{aligned}
$$

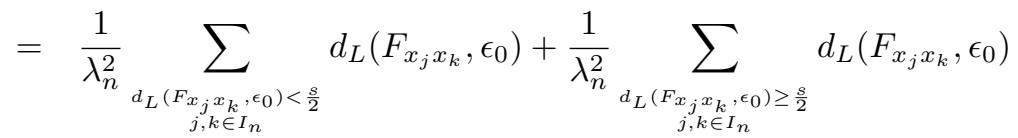

$$
\begin{aligned}
& \leq \frac{s}{2}+\frac{1}{\lambda_{n}^{2}}\left|\left\{(j, k) \in I_{n} \times I_{n}: d_{L}\left(F_{x_{j} x_{k}}, \epsilon_{0}\right) \geq \frac{s}{2}\right\}\right| .
\end{aligned}
$$

Now, since $x$ is strongly $\lambda$-statistically pre-Cauchy, so there exists $n_{0} \in \mathbb{N}$ such that for all $n \geq n_{0}$,

$$
\frac{1}{\lambda_{n}^{2}}\left|\left\{(j, k) \in I_{n} \times I_{n}: d_{L}\left(F_{x_{j} x_{k}}, \epsilon_{0}\right) \geq \frac{s}{2}\right\}\right|<t_{0} .
$$

Thus for all $n \geq n_{0}$, we have

$$
\frac{1}{\lambda_{n}^{2}} \sum_{j, k \in I_{n}} d_{L}\left(F_{x_{j} x_{k}}, \epsilon_{0}\right)<\frac{s}{2}+t_{0}<t .
$$


Hence

$$
\lim _{n \rightarrow \infty} \frac{1}{\lambda_{n}^{2}} \sum_{j, k \in I_{n}} d_{L}\left(F_{x_{j} x_{k}}, \epsilon_{0}\right)=0 .
$$

\section{Strongly Valle-Poussin pre-Cauchy}

Now we treat the condition of the Theorem 3.3 as a new definition of pre-Cauchy and named it strongly Valle-Poussin pre-Cauchy. In particular, when $\lambda_{n}=n$ we call it strongly Cesro pre-Cauchy.

Definition 18. A sequence $x=\left\{x_{k}\right\}_{k \in \mathbb{N}}$ in a PM space $(P, F, \tau)$ is said to be strongly VallePoussin pre-Cauchy if, for every $\varepsilon>0$ there exists $n_{0} \in \mathbb{N}$ such that for all $n \geq n_{0}$,

$$
\frac{1}{\lambda_{n}^{2}} \sum_{j, k \in I_{n}} d_{L}\left(F_{x_{j} x_{k}}, \epsilon_{0}\right)<\varepsilon .
$$

Theorem 4.1. Let $(P, F, \tau)$ be a PM space and $x=\left\{x_{k}\right\}_{k \in \mathbb{N}}$ be a sequence in $P$. Then the sequence $x$ is strongly $\lambda$-statistically pre-Cauchy if and only if $x$ is strongly Valle-Poussin preCauchy.

Proof. Directly follows from the Theorem 3.3.

Theorem 4.2. If $\lim _{n \rightarrow \infty} \frac{\lambda_{n}}{n}=1$, then the notion of strong Valle-Poussin pre-Cauchy implies the notion of strong Cesro pre-Cauchy.

Proof. Let $x=\left\{x_{k}\right\}_{k \in \mathbb{N}}$ be a sequence in a PM space $(P, F, \tau)$ and $x$ be strongly Valle-Poussin pre-Cauchy. Let $\varepsilon>0$ be given. Then there exists $n_{0} \in \mathbb{N}$ such that for all $n \geq n_{0}$, we have

$$
\frac{1}{\lambda_{n}^{2}} \sum_{j, k \in I_{n}} d_{L}\left(F_{x_{j} x_{k}}, \epsilon_{0}\right)<\frac{\varepsilon}{2}
$$

and

$$
\left|\frac{\lambda_{n}}{n}-1\right|<\sqrt{\frac{\varepsilon}{2}} .
$$

Also, we have $d_{L}\left(F_{p q}, \epsilon_{0}\right) \leq 1$ for every $p, q \in P$ and $\lambda_{n} \leq n$ for all $n \in \mathbb{N}$. Thus for all $n \geq n_{0}$, we have

$$
\begin{aligned}
\frac{1}{n^{2}} \sum_{j, k \in[1, n]} d_{L}\left(F_{x_{j} x_{k}}, \epsilon_{0}\right) & =\frac{1}{n^{2}} \sum_{j, k \in\left[1, n-\lambda_{n}\right]} d_{L}\left(F_{x_{j} x_{k}}, \epsilon_{0}\right)+\frac{1}{n^{2}} \sum_{j, k \in I_{n}} d_{L}\left(F_{x_{j} x_{k}}, \epsilon_{0}\right) \\
& \leq \frac{\left(n-\lambda_{n}\right)^{2}}{n^{2}}+\frac{\lambda_{n}^{2}}{n^{2}} \frac{1}{\lambda_{n}^{2}} \sum_{j, k \in I_{n}} d_{L}\left(F_{x_{j} x_{k}}, \epsilon_{0}\right) \\
& <\frac{\varepsilon}{2}+\frac{1}{\lambda_{n}^{2}} \sum_{j, k \in I_{n}} d_{L}\left(F_{x_{j} x_{k}}, \epsilon_{0}\right)
\end{aligned}
$$




$$
\begin{aligned}
& <\frac{\varepsilon}{2}+\frac{\varepsilon}{2} \\
& =\varepsilon .
\end{aligned}
$$

Hence $x$ is strongly Cesro pre-Cauchy.

Corollary 4.3. If $\lim _{n \rightarrow \infty} \frac{\lambda_{n}}{n}=1$, then the notion of strong $\lambda$-statistically pre-Cauchy implies the notion of strong statistically pre-Cauchy.

Proof. Directly follows from the Theorem 3.3 and the Theorem 4.2.

Lemma 4.1. [6] Let $(P, F, \tau)$ be a PM space and $x=\left\{x_{k}\right\}_{k \in \mathbb{N}}$ be a sequence in $P$. If the sequence $x$ is strongly $\lambda$-statistical convergent in $P$, then it has a subsequence $\left\{x_{q_{n}}\right\}_{n \in \mathbb{N}}$ which is strongly convergent to the same limit.

In the very next theorem, we state a sufficient condition for a strongly $\lambda$-statistically preCauchy sequence to be strongly $\lambda$-statistically convergent in a PM space $(P, F, \tau)$.

Theorem 4.4. Let $(P, F, \tau)$ be a PM space and $x=\left\{x_{k}\right\}_{k \in \mathbb{N}}$ be a strongly $\lambda$-statistically preCauchy sequence in $P$. If the sequence $x=\left\{x_{k}\right\}_{k \in \mathbb{N}}$ has a subsequence $\left\{x_{q_{k}}\right\}_{k \in \mathbb{N}}$ which is strongly convergent to $l \in P$ and the following condition holds

$$
0<\liminf _{n \rightarrow \infty} \frac{1}{\lambda_{n}}\left|\left\{q_{k} \in I_{n}: k \in \mathbb{N}\right\}\right|<\infty,
$$

then $x$ is strongly $\lambda$-statistically convergent to $l$.

Proof. Let $t>0$ be given. Then there exists $s>0$ such that for all $u, v, w \in P$, we have

$$
d_{L}\left(F_{u w}, \epsilon_{0}\right)<t \quad \text { whenever } \quad d_{L}\left(F_{u v}, \epsilon_{0}\right)<s \quad \text { and } \quad d_{L}\left(F_{v w}, \epsilon_{0}\right)<s .
$$

Since the subsequence $\left\{x_{q_{k}}\right\}_{k \in \mathbb{N}}$ is strongly convergent to $l$, so there exists $k_{0} \in \mathbb{N}$ such that for all $k \geq k_{0}$, we have $d_{L}\left(F_{x_{q_{k}} l}, \epsilon_{0}\right)<s$. Let $Q=\left\{q_{k}: k \geq k_{0} ; k \in \mathbb{N}\right\}$ and $P(t)=\left\{k: d_{L}\left(F_{x_{k} l}, \epsilon_{0}\right) \geq t\right\}$. Then from (1) we have,

$$
\begin{aligned}
& \frac{1}{\lambda_{n}^{2}}\left|\left\{(j, k) \in I_{n} \times I_{n}: d_{L}\left(F_{x_{j} x_{k}}, \epsilon_{0}\right) \geq s\right\}\right| \\
\geq & \frac{1}{\lambda_{n}^{2}} \sum_{j, k \in I_{n}} \chi_{Q \times P(t)}(j, k) \\
= & \frac{1}{\lambda_{n}}\left|\left\{q_{j} \in Q: q_{j} \in I_{n}\right\}\right| \times \frac{1}{\lambda_{n}}\left|\left\{k \in P(t): k \in I_{n}\right\}\right| .
\end{aligned}
$$

Therefore,

$$
\begin{aligned}
& \lim _{n \rightarrow \infty} \frac{1}{\lambda_{n}^{2}}\left|\left\{(j, k) \in I_{n} \times I_{n}: d_{L}\left(F_{x_{j} x_{k}}, \epsilon_{0}\right) \geq s\right\}\right| \\
\geq & \lim _{n \rightarrow \infty} \frac{1}{\lambda_{n}}\left|\left\{q_{j} \in Q: q_{j} \in I_{n}\right\}\right| \times \lim _{n \rightarrow \infty} \frac{1}{\lambda_{n}}\left|\left\{k \in P(t): k \in I_{n}\right\}\right| .
\end{aligned}
$$

Again since $x$ is strongly $\lambda$-statistically pre-Cauchy, so we have for $s>0$,

$$
\lim _{n \rightarrow \infty} \frac{1}{\lambda_{n}^{2}}\left|\left\{(j, k) \in I_{n} \times I_{n}: d_{L}\left(F_{x_{j} x_{k}}, \epsilon_{0}\right) \geq s\right\}\right|=0 .
$$


Thus

$$
\lim _{n \rightarrow \infty} \frac{1}{\lambda_{n}}\left|\left\{q_{j} \in Q: q_{j} \in I_{n}\right\}\right| \times \lim _{n \rightarrow \infty} \frac{1}{\lambda_{n}}\left|\left\{k \in P(t): k \in I_{n}\right\}\right|=0 .
$$

Now by the given hypothesis, we have $0<\liminf _{n \rightarrow \infty} \frac{1}{\lambda_{n}}\left|\left\{k \in I_{n}: k \in \mathbb{N}\right\}\right|<\infty$.

Therefore,

$$
\lim _{n \rightarrow \infty} \frac{1}{\lambda_{n}}\left|\left\{k \in B(t): k \in I_{n}\right\}\right|=0 .
$$

Thus

$$
\lim _{n \rightarrow \infty} \frac{1}{\lambda_{n}}\left|\left\{k \in I_{n}: d_{L}\left(F_{x_{k} l}, \epsilon_{0}\right) \geq t\right\}\right|=0 .
$$

Hence $x$ is strongly $\lambda$-statistically convergent to $l$.

Note 3. The above result suggests us that there must exist a sequence in a probabilistic metric space which is strongly $\lambda$-statistically pre-Cauchy, however, the sequence is not strongly $\lambda$-statistically convergent.

Example 2. Let $P=\mathbb{R}$ with the usual metric $d$ and $K(x)=1-e^{-x}$. Then $K \in \mathcal{D}^{+}$. Define a function $F: P \times P \rightarrow \mathcal{D}^{+}$by

$$
F(p, q)(t)=F_{p q}(t)=K\left(\frac{t}{d(p, q)}\right)=1-e^{\frac{-t}{|p-q|}} \text { for all } p, q \in P \text { and } t>0
$$

Also, we make the convention that $K(x / 0)=K(\infty)=1$ for $x>0$ and $K(0 / 0)=K(0)=0$. Then $(P, F, \tau)$ becomes a PM space, where $\tau$ is the continuous triangle function. Let $\lambda_{n}=n-3$ for $n>3$ and $\lambda_{n}=1$ for $1 \leq n \leq 3$. Now define a sequence $x$ in $P$ in the following way. For $n, k \in \mathbb{N}$ such that $(n-1) !<k \leq n$ ! we define

$$
x_{k}=\sum_{v=1}^{n} \frac{1}{v} \text {, and let } x=\left\{x_{k}\right\}_{k \in \mathbb{N}} .
$$

Clearly, $x$ has no strongly convergent subsequence by the construction of the sequence. Consequently, by the Lemma $4.1 x$ is not strongly $\lambda$-statistically convergent. However, we show that $x$ is strongly $\lambda$-statistically pre-Cauchy. Let $t_{1}>0$ be given. At first, we define $K_{n}(t)=1-e^{\frac{-t n}{2}}$ for $t>0$. Clearly, $K_{n}(t)$ is a distance distribution function and for $t>0, K_{n}(t)$ weakly converges to $\epsilon_{0}$. Then for that $t_{1}>0$ there exists an positive integer $n_{0}$ such that for all $n \geq n_{0}$ we have $d_{L}\left(K_{n}(t), \epsilon_{0}\right)<t_{1}$. Choose $n>n_{0}$ and $n \geq 4$. Then if $n !<n_{1} \leq(n+1) !$ and $(n-1) !<j, k \leq n_{1}$ then we have, $\left|x_{j}-x_{k}\right|<\frac{2}{n}$. It follows that for $t_{1}>0$ and $n !<n_{1} \leq(n+1)$ !, we have

$$
\begin{aligned}
& \frac{1}{n_{1}^{2}}\left|\left\{(j, k) \in\left[4, n_{1}\right] \times\left[4, n_{1}\right]: d_{L}\left(F_{x_{j} x_{k}}, \epsilon_{0}\right)<t_{1} ; j, k \leq n_{1}\right\}\right| \\
\geq & \frac{1}{n_{1}^{2}}\left[n_{1}-(n-1) !\right]^{2} \\
\geq & {\left[1-\frac{1}{n}\right]^{2} . }
\end{aligned}
$$

Since $\lim _{n \rightarrow \infty}\left[1-\frac{1}{n}\right]^{2}=1$, it follows that $x$ is strongly $\lambda$-statistically pre-Cauchy. 


\section{Acknowledgments}

The authors are thanking both the referees in order of the time for the invaluable contribution that they made to the paper, which shape the paper in its current form. Also, the second author is grateful to the Council of Scientific and Industrial Research (CSIR), India, for his SRF fellowships funding under CSIR-JRF scheme during the preparation of this paper.

\section{References}

[1] J. Connor, J. Fridy and J. Kline, Statistically pre-Cauchy Sequences, Analysis, 14 (1994), $311-317$.

[2] J. Connor, $R$-type summability methods, Cauchy criteria, P-sets and Statistical convergence, Proc. Amer. Math. Soc., 115 (1992), 319-327.

[3] J. Connor, The statistical and strong P-Cesaro convergence of sequences, Analysis, 8 (1988), 47-63.

[4] H. Fast, Sur la convergence statistique, Colloq. Math., 2 (1951), 241-244.

[5] J. A. Fridy, On statistical convergence, Analysis, 5 (1985), 301-313.

[6] P. Malik and S. Das, On strong $\lambda$-statistical convergence of sequences in probabilistic metric (pm) spaces, arXiv preprint arXiv:2007.09173, 2020.

[7] K. Menger, Statistical metrics, Proc. Nat. Acad. Sci., 28 (1942), 535-537.

[8] M. Mursaleen, $\lambda$-statistical convergence, Mathematica Slovaca, 50 (2000), no. 1, 111-115.

[9] T. Šalát, On statistically convergent sequences of real numbers, Mathematica Slovaca, 30 (1980), 139-150.

[10] I. J. Schoenberg, The integrability of certain functions and related summability methods, Amer. Math. Monthly, 66 (1959), 361-375.

[11] C. Şençimen and S. Pehlivan, Strong statistical convergence in probabilistic metric space, Stoch. Anal. Appl., 26 (2008), 651-664.

[12] B. Schweizer and A. Sklar, Statistical metric spaces, Pacific J. Math., 10 (1960), 314-334.

[13] B. Schweizer, A. Sklar and E. Thorp, The metrization of statistical metric spaces, Pacific J. Math., 10 (1960), 673-675.

[14] B. Schweizer and A. Sklar, Probabilistic Metric Spaces, North Holland: New York, Amsterdam, Oxford, 1983.

[15] R. M. Tardiff, Topologies for Probabilistic Metric spaces, Pacific J. Math., 65 (1976), 233-251.

[16] E. Thorp, Generalized topologies for statistical metric spaces, Fundamenta Mathematicae, 51 (1962), 9-21. 
Argha Ghosh Department of Mathematics, Brainware University, Barasat, Kolkata-700125, West Bengal, India.

E-mail: buagbu@yahoo.co.in

Samiran Das Department of Mathematics, The University of Burdwan, Golapbag, Burdwan713104, West Bengal, India.

E-mail: das91samiran@gmail.com 